\title{
A genome-wide association study using a Vietnamese landrace panel of rice (Oryza sativa) reveals new QTLs controlling panicle morphological traits
}

Kim Nhung TA ${ }^{1,6}$, Ngan Giang KHONG ${ }^{1,7}$, Thi Loan HA ${ }^{1}$, Dieu Thu NGUYEN ${ }^{1}$, Duc Chung MAl' ${ }^{1}$, Thi Giang HOANG ${ }^{1}$, Thi Phuong Nhung PHUNG ${ }^{1}$, Isabelle BOURRIE ${ }^{2}$, Brigitte COURTOIS ${ }^{3}$, Thi Thu Hoai TRAN ${ }^{4}$, Bach Yen DINH ${ }^{4}$, Tuan Nghia LA ${ }^{4}$, Nang Vinh DO', Michel LEBRUN ${ }^{1,5}$, Pascal GANTET ${ }^{1,2}$ and Stefan JOUANNIC ${ }^{1,2^{*}}$ (D)

\begin{abstract}
Context: Yield improvement is an important issue for rice breeding. Panicle architecture is one of the key components of rice yield and exhibits a large diversity. To identify the morphological and genetic determinants of panicle architecture, we performed a detailed phenotypic analysis and a genome-wide association study (GWAS) using an original panel of Vietnamese landraces.

Results: Using a newly developed image analysis tool, morphological traits of the panicles were scored over two years: rachis length; primary, secondary and tertiary branch number; average length of primary and secondary branches; average length of internode on rachis and primary branch. We observed a high contribution of spikelet number and secondary branch number per panicle to the overall phenotypic diversity in the dataset. Twenty-nine stable QTLs associated with seven traits were detected through GWAS over the two years. Some of these QTLs were associated with genes already implicated in panicle development. Importantly, the present study revealed the existence of new QTLs associated with the spikelet number, secondary branch number and primary branch number traits.

Conclusions: Our phenotypic analysis of panicle architecture variation suggests that with the panel of samples used, morphological diversity depends largely on the balance between indeterminate vs. determinate axillary meristem fate on primary branches, supporting the notion of differences in axillary meristem fate between rachis and primary branches. Our genome-wide association study led to the identification of numerous genomic sites covering all the traits studied and will be of interest for breeding programs aimed at improving yield. The new QTLs detected in this study provide a basis for the identification of new genes controlling panicle development and yield in rice.
\end{abstract}

Keywords: Rice, GWAS, Panicle, Architecture, Yield, Image analysis, Vietnam

\section{Background}

Rice (Oryza sativa L.) is one of the most important food crops for more than half of the world's population and is also considered as a model species for grasses for important agronomic traits. Due to the rapid growth of the world's population as well as increasing urbanization

\footnotetext{
* Correspondence: stephane.jouannic@ird.fr

'LMI RICE, University of Montpellier, IRD, CIRAD, USTH, National Key

Laboratory for Plant Cell Biotechnology, Agronomical Genetics Institute, Hanoi, Vietnam

${ }^{2}$ UMR DIADE, University of Montpellier, IRD, Montpellier, France

Full list of author information is available at the end of the article
}

and climatic changes, higher or at least sustainable rice yield is urgently required to meet world food demands. Rice yield is a complex agronomic trait that is directly determined by three component traits: the number of panicles per plant, the number of grains per panicle and grain weight $[1,2]$. While grain weight is determined by two components, grain size (length, width and thickness) and the degree of grain filling, the number of panicles is dependent on tillering ability. The number of grains per panicle is dependent on the panicle architecture, which consists of a series of branches of different orders:

(c) The Author(s). 2018 Open Access This article is distributed under the terms of the Creative Commons Attribution 4.0 International License (http://creativecommons.org/licenses/by/4.0/), which permits unrestricted use, distribution, and 
rachis, primary branches, secondary branches, potentially tertiary branches and finally spikelets. In rice, one spikelet meristem produces one floral meristem; therefore, the number of spikelet meristems will determine the number of grains per panicle.

By analysing the diversity of yield-related traits in rice, many studies aiming at creating new varieties with higher yield have been carried out [1]. Over several decades, quantitative trait locus (QTL) mapping using bi-parental populations of $O$. sativa has allowed the identification of QTLs related to characters such as panicle branching or size (e.g. Gn1a, DEP1, IPA1/WFP), grain weight or size (e.g. GS3, GW2, qSW5/GW5) and grain filling (e.g. GIF1), thus revealing a large number of genes governing yield components [1-3]. The identification of other QTLs has shown that yield potential can also be influenced indirectly by factors relating to the physiological state of the plant. An example is the GPS (GREEN FOR PHOTOSYNTHESIS) QTL that influences photosynthesis rate through the regulation of carboxylation efficiency $[4,5]$. Some specific alleles of the genes corresponding to these yield-related QTLs were selected during domestication and/or breeding programs for high yields.

More recently, genome-wide association studies (GWAS), applied to large panels of rice varieties, emerged as a more powerful approach to increase coverage of natural variation and the number of significant loci, especially for complex agronomic traits [6]. The development of New Generation Sequencing (NGS)-based technologies allows the study of genotypically wide panels with a high density of markers covering the entire genome. GWAS analyses were conducted on different panels of rice accessions, leading to the description of genomic regions of interest related to various agronomic traits such as leaf traits, primary metabolism, plant height, flowering time, grain quality, grain size, grain coloration and physiological features, salt and drought tolerance, blast resistance, nematode tolerance, aluminium tolerance, and root system architecture [7-16]. These studies highlighted not only previously characterized QTLs but also numerous new QTLs and candidate genes, providing a valuable resource for rice genetics research as well as genetic markers for breeding [7, 17]. Recently, three GWAS studies were published relating to morphological components of panicle architecture using different panels of genotypes [18-20]. These analyses led to the identification of numerous genomic sites of interest. Only a fraction of these sites were associated with genes already known to be involved in panicle development, indicating that a number of as yet unknown factors may be involved in the determination of panicle architecture. Moreover, these analyses illustrated the impact of the environment on the identified genomic sites, supporting the view that panicle architecture depends not only on genetic diversity but also the environment.
In this study, as a means of further dissecting the morphological components of panicle architecture, a genome-wide association study of panicle morphological traits was performed on a panel of Vietnamese landraces using an image-based analysis tool [21, 22]. Vietnam possesses a huge diversity of traditional rice varieties due to its geographical situation and range of ecosystems [23]. Vietnamese resources still constitute a largely untapped and highly valuable genetic resource for local breeding programs. In addition, only a small fraction of the rice genetic diversity of Vietnam has been exploited in previous association studies based on worldwide sampling. The phenotypic analysis of the Vietnamese panel we used indicated that the number of secondary branches is the main contributor to variation in spikelet number per panicle. Several QTLs associated with spikelet number, secondary branch number and primary branch number were identified in the full panel as well as in the indica and japonica subpanels. These results will be useful for the exploitation of these varieties in local breeding programs and will provide new knowledge for understanding the complexity of the genetic basis of panicle architecture.

\section{Methods \\ Plant material and genotyping}

A population of 159 traditional rice varieties representing the diversity of $O$. sativa species in Vietnam and three reference varieties (Nipponbare, IR64 and Azucena) was used for GWAS. This population was obtained from a Vietnamese landrace core-collection established from 2014 and genotyped using 241 Diversity Array Technology (DArT) markers and 25,971 Genotyping By Sequencing (GBS)-derived Single Nucleotide Polymorphim (SNP) markers [22]. The population was structured into two sub-panels (93 indica accessions distributed within 6 sub-groups and 63 japonica accessions distributed within 4 sub-groups) and three admixed accessions that were determined using STRUCTURE software v2.3.4 [24]. The information related to these varieties is presented in Additional File 1: Table S1 and are detailed in [22].

\section{Phenotypic analysis}

Phenotyping for GWAS analysis was performed in field conditions near Hanoi at the Plant Resource Center located at An Khanh, Hoai Duc $\left(21^{\circ} 00^{\prime} 01^{\prime \prime} \mathrm{N}\right.$ and $105^{\circ}$ $72^{\prime} 55^{\prime \prime}$ E) and Van Giang Agricultural Station located at Van Giang, Hung Yen $\left(20^{\circ} 90^{\prime} 42^{\prime \prime} \mathrm{N}\right.$ and $105^{\circ} 94^{\prime} 78^{\prime \prime}$ E) during the wet season of 2014 and 2015, respectively. In both field experiments, seeds were sown at the same period. After transplanting at 2 weeks after sowing, the varieties were grown in lowland conditions in $1 \mathrm{~m}^{2}$ plots of 25 plants each. The experimental design was an alpha-lattice with 2 replicates [25]. A block factor of 9 
was introduced to check for possible environmental variations within replicates, a single block containing 18 accessions (i.e. 18 plots).

About twenty days after the heading date, the three main panicles from three randomly chosen plants per variety per replicate were collected (i.e. 9 panicles/accession/replicate). Each panicle was spread out and fixed on white paper using tape. A total of 2916 panicles per season were analyzed using the P-TRAP software [21]. The quantified panicle traits included rachis length (RL), primary branch number $(\mathrm{PBN})$, primary branch average length (PBL), primary branch internode average length (PBintL), secondary branch number (SBN), secondary branch average length (SBL), secondary branch internode average length (SBintL) and spikelet number $(\mathrm{SpN})$ (Fig. 1a and Additional file 1: Table S1). Additional traits such as flowering date (FT), tiller number (TN) and efficient tiller number (eTN) were also recorded (Additional file 1: Table S1).

Panel statistics were calculated and statistical analysis of variance were performed using functions in $\mathrm{R}$ software. Analysis of variance (ANOVA) was applied, taking into consideration all factors (replicates, blocks within replicates and varieties) as fixed effects. Mean values were adjusted for variety, replicate and block factors. Trait heritability was computed using the variety effect based on the variance among phenotypic measurements between the two replicates of the population. A Shapiro test was used to determine if a dataset was well modeled by a normal distribution. The corrplot, ade 4 and devtool packages were used to analyze the phenotypic correlation between traits and the organization of phenotypic variability, while linear regression analysis was used to obtain the coefficients of determination between PBN and $\mathrm{SpN}$ on the one hand, and $\mathrm{SBN}$ and $\mathrm{SpN}$ on the other hand. All statistical tests, clustering analysis, heat-map and principal component analyses (PCA) of the phenome dataset were performed using $\mathrm{R}$ software.

\section{Genome-wide association analysis}

The entire genotypic panel, including 159 accessions with 26,212 markers, was analysed by applying a mixed linear model (MLM) according to [26]. The kinship matrix was established using the pairwise Identity by State (IBS) method proposed by Tassel v5.2.8 [27]. The structure matrix of the panels was determined by running a principal component analysis (PCA). The first two PCA axes were retained, respectively, and the scores of the accessions on these axes were used as the structure matrix. Three independent analyses were conducted using the full panel, the indica sub-panel and the japonica sub-panel respectively. Quantile-Quantile plots (QQ plots) and Manhattan plots with the threshold lines were performed using the QQman $\mathrm{R}$ package. A significance threshold of $P<0.001$, based on the number of detected sites, was considered as corresponding to a significant association considering the globally low $p$-values. Only associations detected at $P<0.001$ over the two years were considered as significant for further analysis.

Linkage disequilibrium (LD) between SNPs in the panel was evaluated by computing the $r^{2}$ values between pairs of SNP markers using Tassel v5.2.8 on a chromosome basis. The LD heatmaps surrounding peaks in the GWAS were constructed using the LDheatmap R package and the candidate regions were defined by considering LD blocks with $r^{2}>0.6$. On one hand, the significant markers of each LD block were used to define haplotypes using $\mathrm{R}$ software and then compare with the phenotype of each haplotype. On the other hand, genes and miRNA loci in LD regions were identified using MSU7, Rap_db database and mirBase v21 annotations (http://rice.plantbiology.msu.edu, http:// www.gramene.org, http://www.mirbase.org, respectively). The qTARO (http://qtaro.abr.affrc.go.jp) and funRiceGenes databases (http://funricegenes.ncpgr.cn) were used to identify QTLs and to functionally characterize genes that overlapped with the LD regions associated with significant markers.

\section{Results \\ Panicle architecture trait variation and heritability in the Vietnamese landrace panel}

A Vietnamese landrace population [22] was phenotyped over two wet seasons (June to November 2014 and 2015) in lowland irrigated conditions in North Vietnam near Hanoi. Based on image analysis, a total of 9 panicle traits were scored (Fig. 1a, b and Additional file 1: Table S1). Each trial was replicated two times. Statistical analysis revealed that differences between the two replicates of the same year were not significant for any panicle traits $(P>0.001$ at $1 \%$ threshold) (Table 1). Calculation of heritability scores showed that the scores of almost all panicle traits were similar and with high values $\left(\mathrm{H}^{2}>0.85\right)$ for the two years, except for TBN which showed the lowest heritability score (0.21 in 2014 and 0.43 in 2015) and the highest variation with a coefficient of variation (CV) of the trait of over 35\% with no significant genotypic effect (Table 1). This suggested that TBN depended more on environment than on genetic background. Consequently, this trait was not considered for the GWAS study. For other traits, secondary branch internode length and secondary branch number (SBintL, SBN) also displayed a wide phenotypic variation with a CV of over $25 \%$ while the CVs of other traits such as the length and number of primary branches and spikelets (SBL, PBL, PBN, SpN) were around 14-20\% (Table 1).

Comparison of panicle traits between the indica and japonica subpanels for each year indicated that the number of spikelets $(\mathrm{SpN})$ as well as the number of secondary (SBN) and primary branches $(\mathrm{PBN})$ were similar between the two subpanels (Additional file 2: Figure S1). This similarity indicated that population structure and phenotypic diversity 

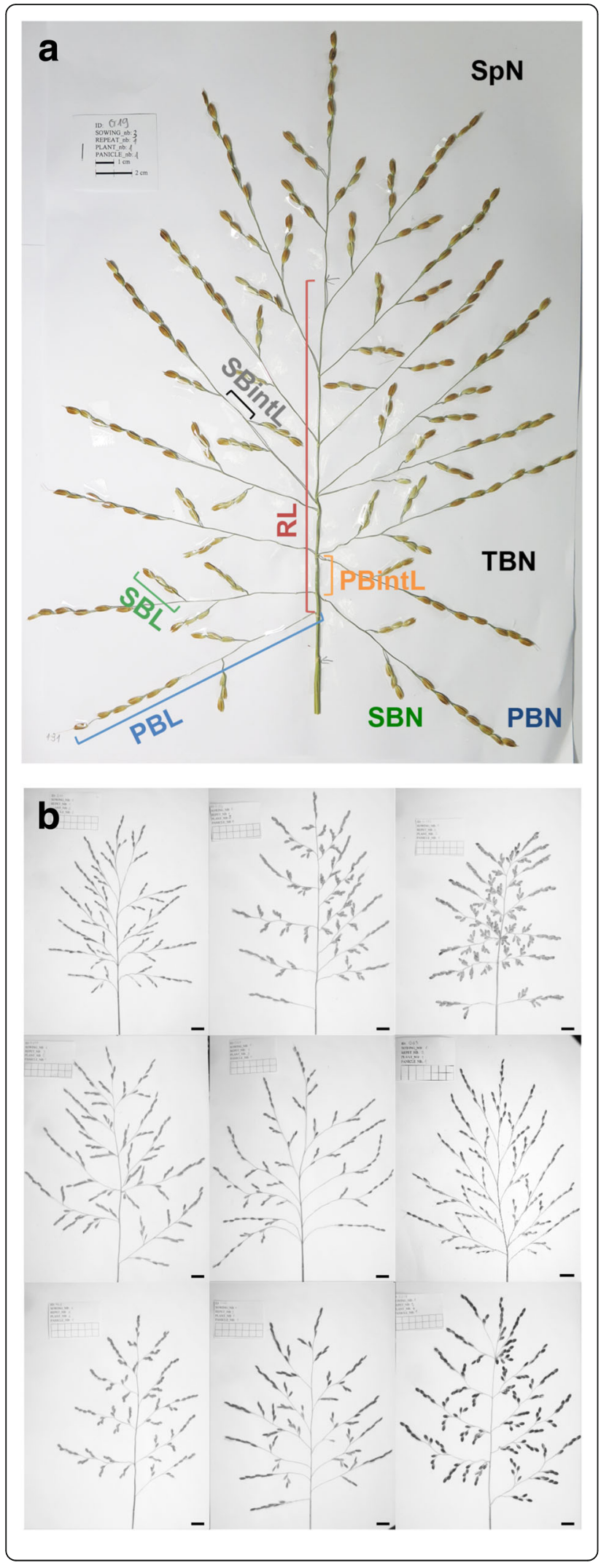

Fig. 1 Panicle phenotyping. (a) Spread panicle with the quantified morphological traits using P-TRAP software. SpN: spikelet number; SBN: secondary branch number; PBN: primary branch number; TBN: tertiary branch number; SBintL: secondary branch internode average length; PBintL: primary branch internode average length; PBL: primary branch average length; SBL: secondary branch average length; RL: Rachis length; (b) Illustration of panicle architecture diversity in the Vietnamese landrace panel. Scale bar: $2 \mathrm{~cm}$

were not correlated for these traits, leading in turn to the conclusion that this population was suitable for GWAS analysis of the traits in question. However, rachis lengths (RL) and primary internode average lengths (PBintL) within the japonica subpanel were found to be greater than for those of the indica subpanel (Additional file 2: Figure S1). In parallel, secondary branch internode average length (SBintL) was found to be shorter in the japonica subpanel than in the indica subpanel. Overall, japonica accessions in this population tend to have larger panicles than in the indica accessions. A comparison between the two years revealed a switch in the distribution of mean values for all traits, with significantly higher values in 2015 than in 2014 (Fig. 2). Overall, panicles in the 2015 experiment were larger and bore more spikelets than those of the 2014 experiment. However, traits relating to structure length were more impacted than those relating to structure numbers.

\section{Correlations between panicle architecture traits}

Studies of phenotypic correlation and principal component analyses (PCA) between all traits revealed similar patterns over the two years (Fig. 3). Firstly, number-related traits $\mathrm{SpN}, \mathrm{PBN}$ and SBN were highly positively correlated with each other (Fig. 3a and b), especially between SpN and SBN with a correlation value of over 0.94 for both years. The

Table 1 Phenotype variation and broad sense heritability $\left(\mathrm{H}^{2}\right)$ for each panicle architecture trait in 2014 and 2015

\begin{tabular}{|c|c|c|c|c|c|c|}
\hline & 2014 & & & 2015 & & \\
\hline Traits & Mean & CV & $\mathrm{H}^{2}$ & Mean & $C V$ & $\mathrm{H}^{2}$ \\
\hline SpN & 180.9 & 21.78 & 0.91 & 216.1 & 22.57 & 0.89 \\
\hline PBN & 12.3 & 15.02 & 0.94 & 13.6 & 14.23 & 0.93 \\
\hline SBN & 34.1 & 26.18 & 0.91 & 40.7 & 25.47 & 0.88 \\
\hline TBN & 0.1 & 154.45 & 0.21 & 0.6 & 193.97 & 0.43 \\
\hline PBL & 11.3 & 13.64 & 0.94 & 16.5 & 14.07 & 0.93 \\
\hline SBL & 2.6 & 15.01 & 0.91 & 3.6 & 14.92 & 0.96 \\
\hline $\mathrm{RL}$ & 19.7 & 17.76 & 0.90 & 29.4 & 18.58 & 0.92 \\
\hline PBintL & 1.8 & 23.17 & 0.93 & 2.4 & 22.04 & 0.96 \\
\hline SBintL & 0.8 & 41.24 & 0.91 & 1.1 & 36.71 & 0.92 \\
\hline
\end{tabular}

$S p N$ spikelet number, $P B N$ primary branch number, $S B N$ secondary branch number, $T B N$ tertiary branch number ,PBL primary branch average length, $S B L$ secondary branch average length, $R L$ Rachis length, PBint $L$ primary branch internode average length, SBintL secondary branch internode average length, Mean Mean value of traits of 2 replicates, $C V$ coefficient of variation of the trial 


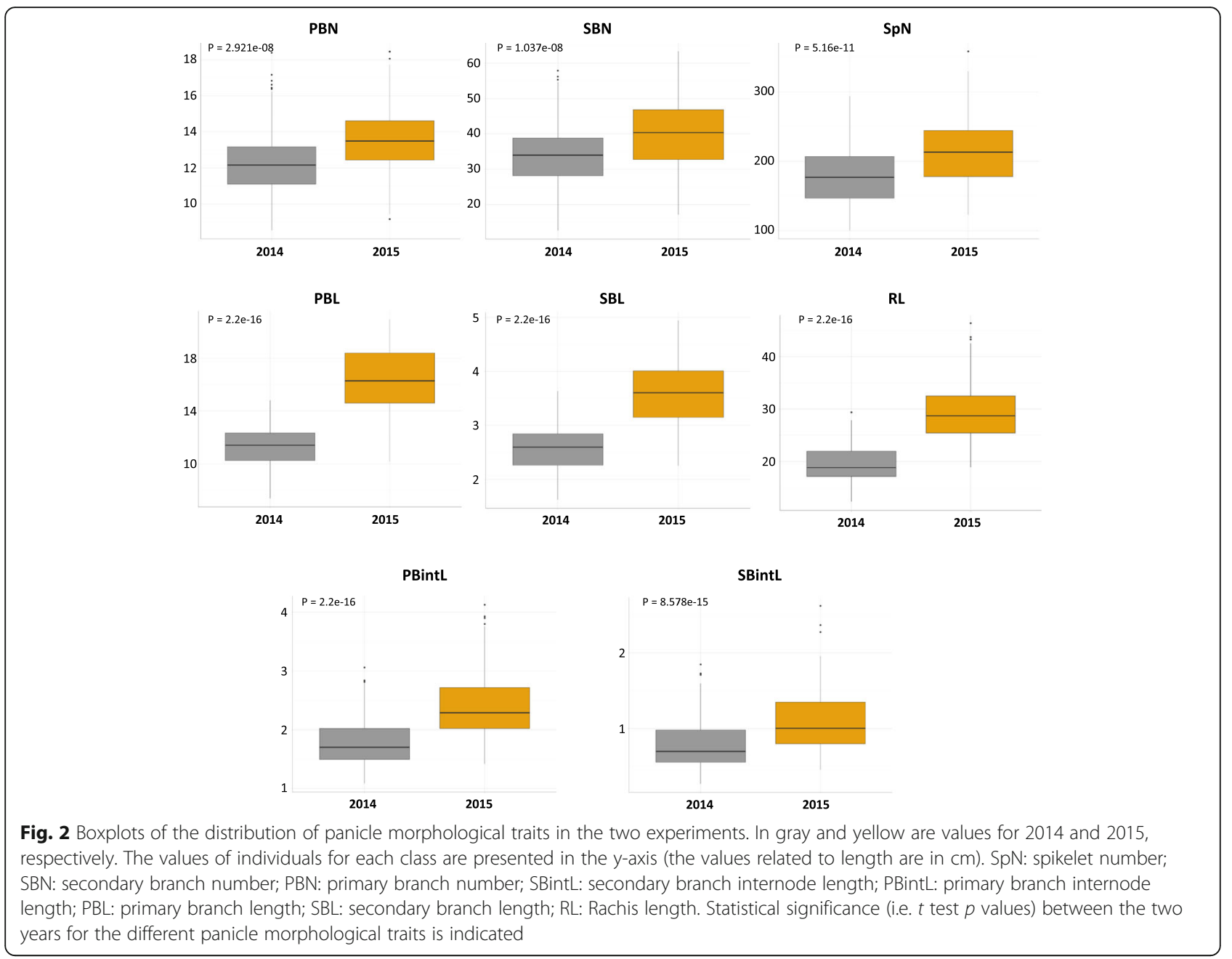

correlation between SpN and SBN was observed not only in the full panel, but also in the indica and japonica subpanels (Additional file 2: Figure S1 and Additional file 3: Figure S2). As secondary branches derive from primary branches, it is to be expected that a strong positive correlation is observed between the numbers of primary and secondary branches (Fig. 3a and b). Secondly, primary and secondary branch lengths were highly correlated with each other and were also correlated with rachis length albeit at a lower level, suggesting that these morphological components are determined by a globally regulated elongation process in the panicle. A low positive correlation was also observed between the average length of primary branches and the number of secondary branches, indicating that longer primary branches produce more secondary branches. In contrast, the length of the branches and their number were not correlated (i.e. PBN vs. PBL and SBN vs. SBL). In the same way, as expected, a negative correlation was observed between the numbers of primary or secondary branches and the average length of internodes (i.e. PBN vs. PBintL and SBN vs. SBintL). Finally, rachis lengths showed a positive correlation with all number-related traits (PBN, SBN and SpN) in addition to branch length (PBL and SBL) and PBintL. Additional traits were scored in the field such as flowering time (FT) in 2014 and 2015, and plant height $(\mathrm{PH})$, tiller number $(\mathrm{TN})$ and efficient tiller number (eTN) in 2015 only (Additional file 1: Table S1 and Additional File 3: Figure S2). A low positive correlation between FT and $\mathrm{PH}$ was observed over the two years. These two traits were positively correlated with panicle number traits but negatively correlated at a low level to panicle length traits over the two years. TN and eTN were highly correlated as expected but negatively correlated with panicle number traits and RL.

Principal component analysis (PCA) revealed that the investigated traits could be resolved into two groups: number-related traits (PBN, SBN, $\mathrm{SpN})$ and length-related traits (RL, PBL, SBL, PBintL, SBinL), with the first two PCA axes explaining about $60 \%$ of panicle trait variation (Fig. 3c and d). Spikelet number per panicle was the main trait contributing to the diversity observed in this population with a high correlation with 


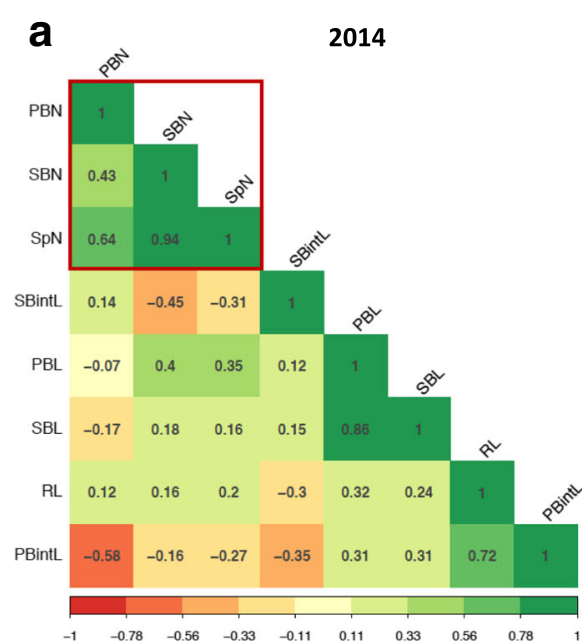

C

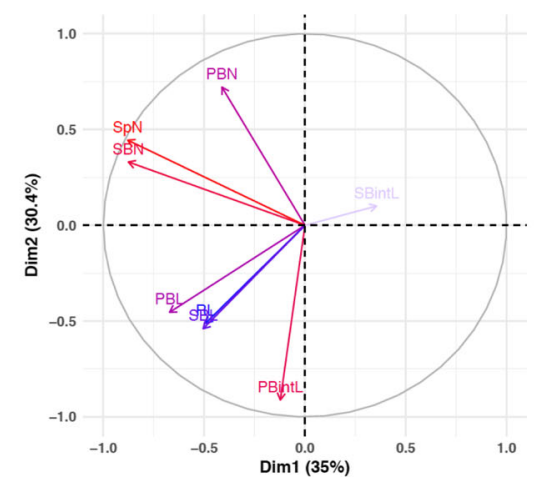

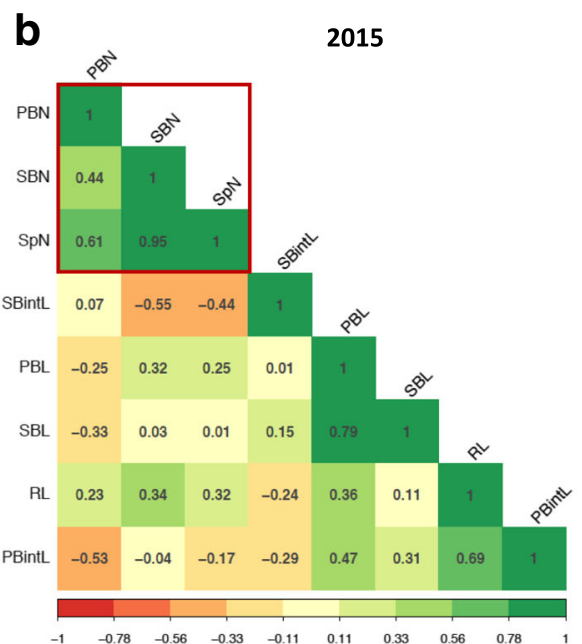

d

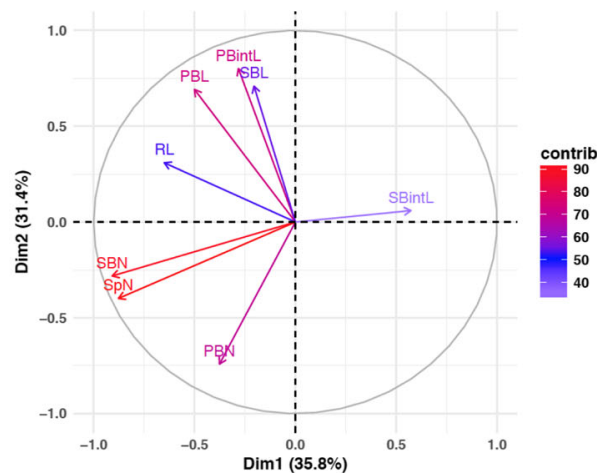

Fig. 3 Correlation between panicle morphological traits of the full panel in the two experiments. (a-b) Correlation plots of panicle morphological traits for 2014 and 2015, respectively. (c-d) Principal component analysis (PCA) of panicle morphological traits for 2014 and 2015, respectively

SBN and intermediate correlation with PBN for the full panel as well as for the individual indica and japonica subpanels (Fig. 3c, d and Additional file 3: Figure S2). The adjusted $\mathrm{R}^{2}$ of linear regression analysis showed that the distribution of SBN explained 89 to $91 \%$ of SpN variation, while $\mathrm{PBN}$ variation explained only 37 to $42 \%$ of $\mathrm{SpN}$ variation (Fig. 4 and Additional file 4: Figure S3).

In addition, to establish a global phenotypic map of panicle traits for the two subpanels (indica and japonica), a clustering analysis was performed on the phenome dataset (Additional file 5: Figure S4). In a similar way to PCA, this analysis revealed distinct clades between the traits relating to structure numbers (PBN, $\mathrm{SBN}, \mathrm{SpN}$ ) and the traits relating to structure length (RL, PBL, SBL, PBintL, SBintL). Moreover this analysis confirmed that the clustering of the population based on panicle morphological traits was not strictly related to the genetic origin of the accessions (indica vs. japonica), with the exception of some clades (Additional file 5: Figure S4). This suggests that the full panel is suitable for GWAS for most of the traits studied here.

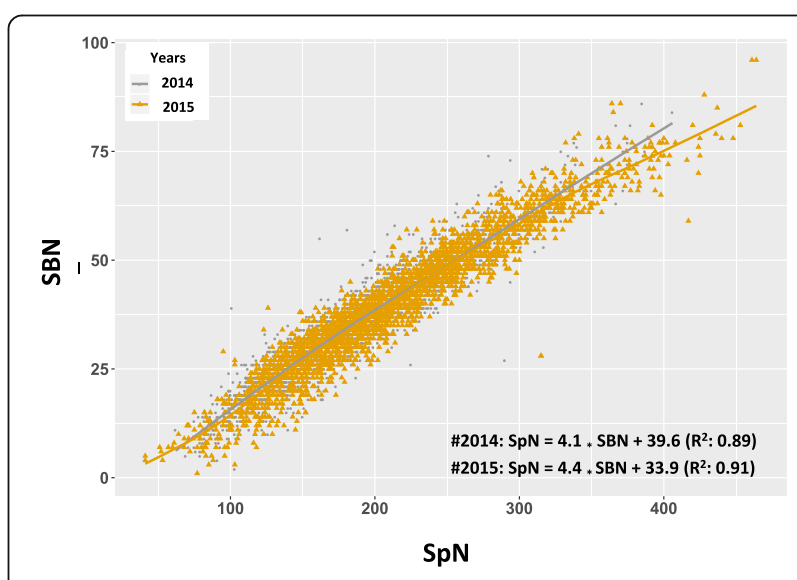

Fig. 4 Correlation between spikelet number and secondary branch number in the full panel. In grey and yellow are values for 2014 and 2015, respectively. SpN: spikelet number. SBN: secondary branch number. The adjust $R^{2}$ values of linear regression are indicated 


\section{GWAS for panicle architecture traits in the Vietnamese landrace panel}

A set of 26,212 SNPs covering all the genome was selected from an initial genetic characterization of this Vietnamese landrace population [22]. This set was used on our panel of 159 accessions. We used a mixed linear model (MLM) to detect association signals for 8 traits: SpN, PBN, SBN, RL, PBL, SBL, PBintL and SBintL. In our study, the correlation between panicle traits and flowering date was not high (Additional file 3: Figure S2). Consequently, no correction/ normalisation based on flowering date was carried out in our study. A total of 508 and 421 significant SNPs $(P<$ 0.001 ) were detected for the 8 traits in 2014 and 2015 respectively (Additional file 6: Table S2). One hundred and five of these significant SNPs were stable over the two years. Manhattan plots and Quantile-Quantile plots for all traits over the two years are shown in Additional file 7: Figure S5. This analysis led to the identification of 29 QTLs detected for the same trait(s) over the two years (Table 2; see Additional file 8: Table S3 for the details). Corresponding genomic segments were defined according to the LD block size around the significant marker(s). For a single significant marker within a low LD block, a segment of $100 \mathrm{~Kb}$ around the marker $( \pm 50 \mathrm{~Kb})$ was arbitrarily selected.

A total of 6 stable QTLs were detected for SpN, 9 for branch number (8 for PBN, 1 for $\mathrm{SBN}$ ), and 17 for length-related traits ( 1 for PBL, 1 for SBL, 4 for RL, 10 for PBintL and 1 for SBintL) (Table 2). Although a strong correlation was observed between $\mathrm{SpN}$ and branch number traits, especially SBN, only 2 of the 6 stable QTLs relating to SpN colocalized with other number-related traits (PBN and SBN) on chromosomes 1 and 2, respectively (QTL_6 and QTL_9, respectively in Table 2). However, QTL_6 was defined by a single significant marker, in contrast to QTL_9 which was defined by 9 significant markers (Table 2). Two other co-localizations were observed for RL and PBLintL on one hand, and for PBN and PBL on the other (QTL_14 and QTL_19, respectively in Table 2). No co-localizations were observed for PBL and SBL, nor for PBN and SBN. Of the 29 QTLs, 17 (59\%) and 7 (24\%) were detected specifically in the full panel and in the indica subpanel, respectively (Table 2). The 5 remaining QTLs were detected in the full panel and also in one of the two subpanels. Of the 13 QTLs relating to number traits detected over the two years, 8 were supported by more than one significant marker in the LD block (QTL_1, QTL_4, QTL_5, QTL_7, QTL_9, QTL_10, QTL_11 and QTL_24) (Table 2). Polymorphism combination analysis for these QTLs using the significant SNPs detected showed that the high $\mathrm{SpN}$ values were associated with the ATG haplotype in QTL_4, and with the ATATAAAT haplotype in QTL_9 (as SBN values for this QTL). The high PBN values were associated with the AAAAAAATAT, ATAAAAA and ATTAT haplotypes in the QTL_1, QTL_5 and QTL_24, respectively (Fig. 5). For QTL_7, QTL_10 and QTL_11, it was not possible to assign a specific haplotype to the high values of associated traits. QQ-plots and Manhattan plots over the 12 chromosomes for SpN, PBN and SBN are shown in Fig. 6, as well as LD heatmap plots for QTL_4, QTL_5, QTL_9 and QTL_24 illustrating the colocalization of GWAS sites and genes involved in panicle development and the colocalization of a GWAS site for both SpN and SBN.

In addition, twenty-one genomic regions were detected significantly over the two years but were associated with different traits (QTL_30 to QTL_50, Additional file 8: Table S3). In this context, 4 additional QTLs were detected for SpN, 10 for PBN, 4 for SBN, 3 for PBL, 3 for SBL, 10 for RL, 10 for PBintL and 5 for SBintL.

\section{Co-localized QTLs and functionally characterized genes}

Most of the QTLs detected in this study co-localized with previously reported QTLs identified in bi-parental population studies and from other GWAS analyses relating to panicle and yield traits (Table 2; see Additional file 8: Table S3 for detailed information). Thirty QTLs of the 50 detected in our study overlapped with previously reported GWAS sites relating to panicle architecture (Additional file 8: Table S3) [18, 19]. Of the 25 co-localized sites common to our study and [18], only 3 sites shared comparable/similar traits (namely QTL_18 and QTL_38 for PBintL, and QTL_39 for PBN) (Additional file 8: Table S3). Similarly, only 4 sites co-localized with GWAS-derived QTLs from an indica panel phenotyped using the same image analysis software, but not for the same panicle trait [19] (Additional file 8: Table S3). In the later analysis, two QTLs displaying co-localization for SpN and SBN traits, one on chromosome 2 (q-9) and one on chromosome 11 (q-46), were reported but none of them co-localized with QTL_9 associated with the two traits in our study [19].

Co-localization with QTLs identified in bi-parental populations were observed for the same traits as for QTL_2 and QTL_13 for SpN trait and QTL_19 for the PBN trait (Additional file 8: Table S3). QTLs identified in bi-parental populations that related to other traits co-localized with GWAS-derived QTLs detected in our study. Among these, we observed co-localization of QTLs relating to the number of vascular bundles, root branching, chlorophyll content or flag leaf length with GWAS QTLs relating to primary branch number, spikelet number or rachis length (Additional file 8: Table S3). In the same way, co-localization was observed with GWAS QTLs relating to the root system that were identified using the same genetic population (Additional file 8: Table S3).

Of the 29 QTLs that displayed stability over the two years, 7 were associated with genes previously known to control panicle development and 3 additional QTLs were found to be associated with genes having an annotation suggestive of a role in the control of development (Table 2, Fig. 6 and Additional file 8: Table S3). In addition several annotated microRNAs were localized in the stable QTLs relating to 


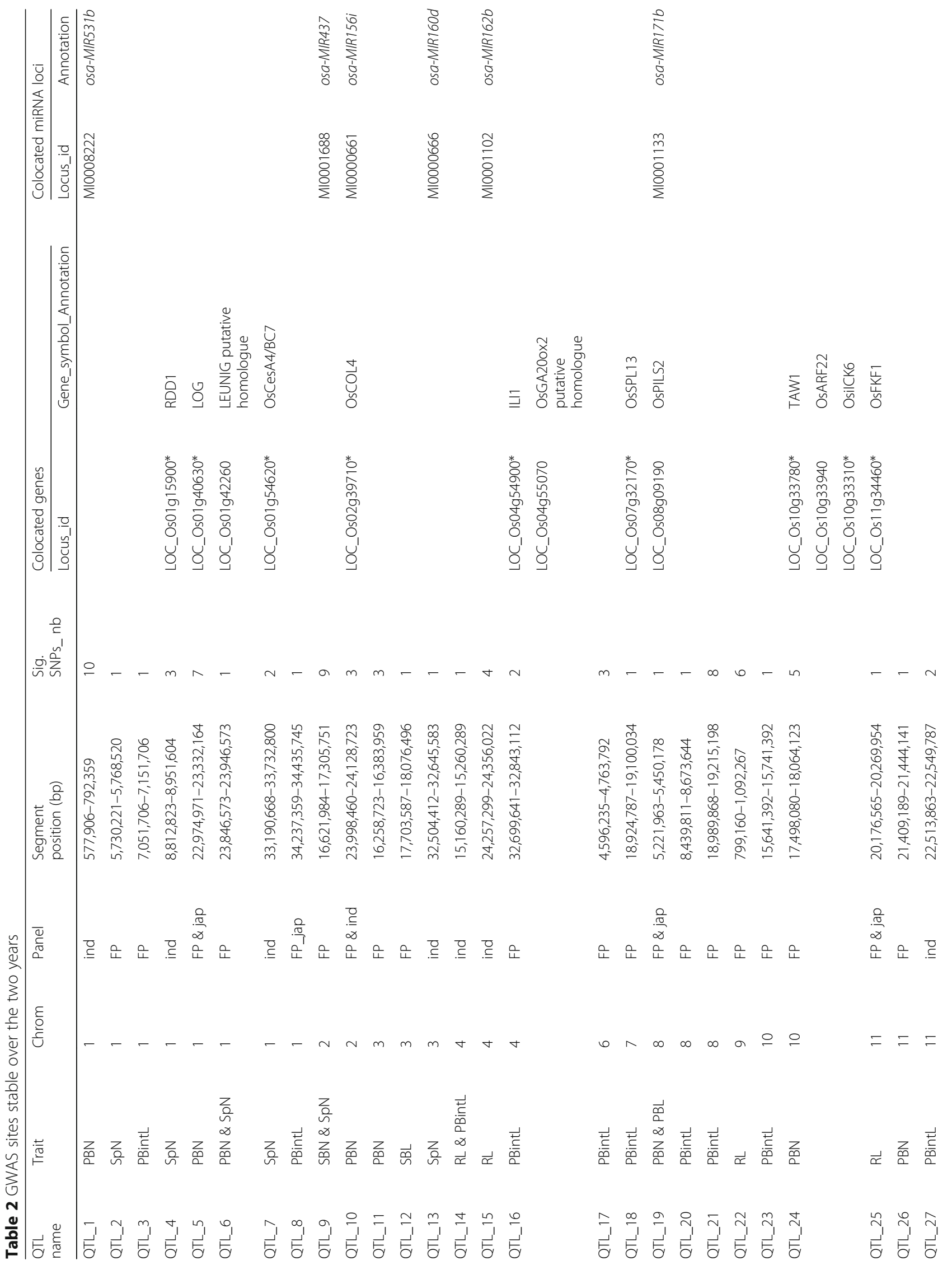




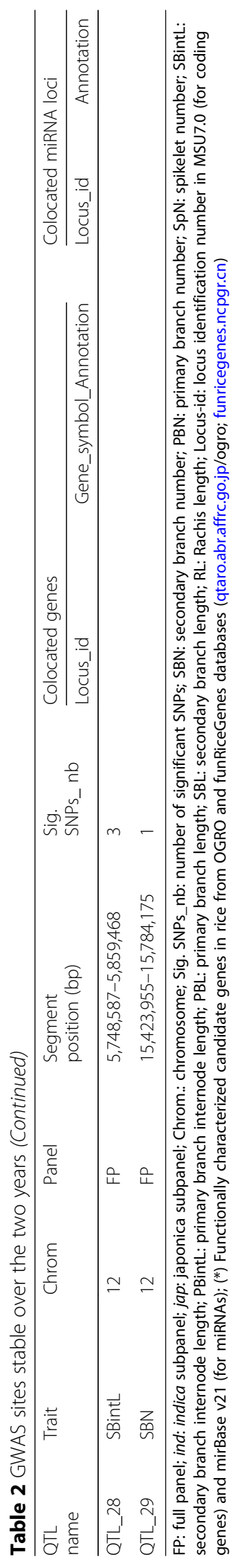



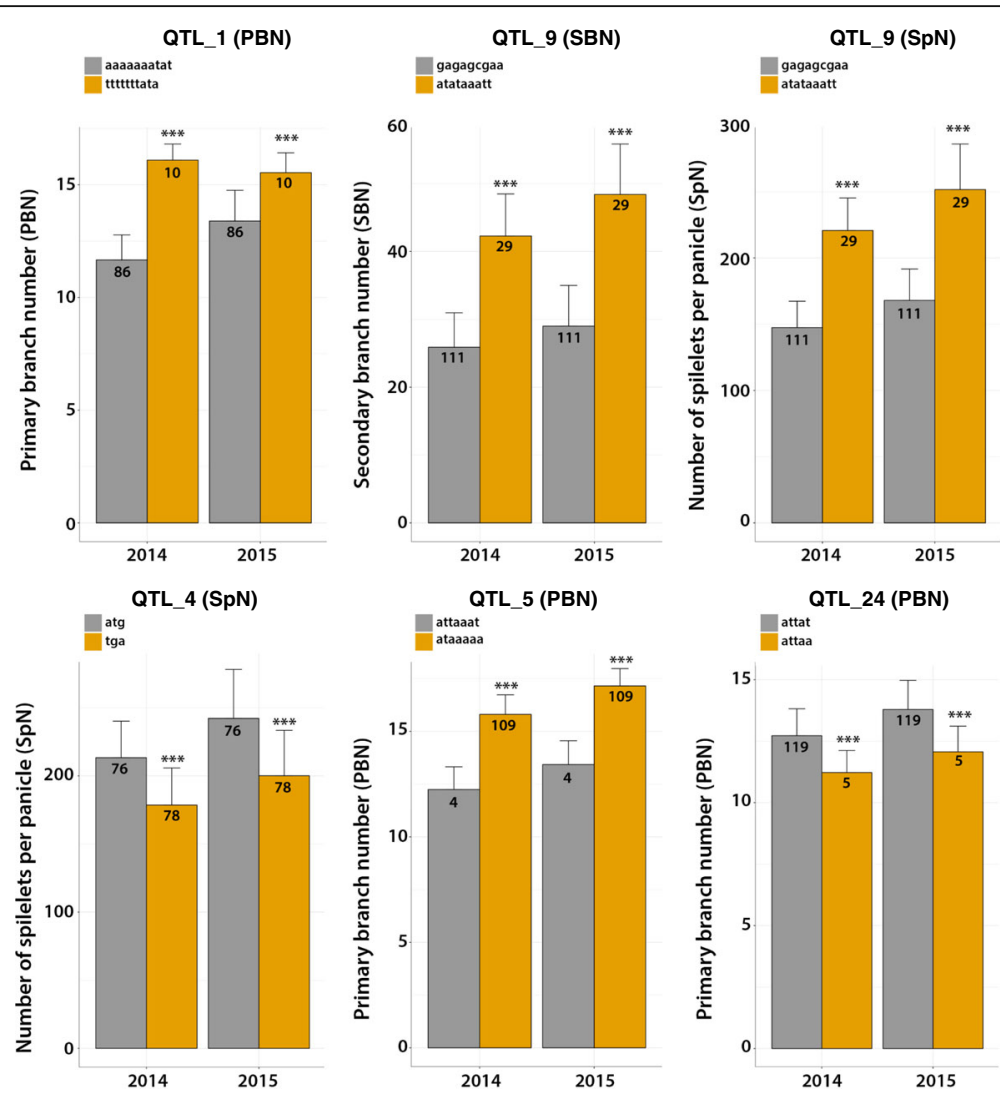

Fig. 5 Allelic combination for QTL_1, QTL_4, QTL_5, QTL_9 and QTL_24 and their effect on the number of spikelets per panicle, secondary branch number and primary branch number. ${ }^{* *}$ Indicates significant differences at $p<0.001$ between allelic combination for each experiment. The two main haplotypes are represented. The number of accessions for each haplotype is indicated $(n)$

panicle traits (Table 2; Additional file 8: Table S3). No candidate genes could be identified for QTL_9 (i.e. QTL associated to SpN and SBN traits on chromosome 2) on the basis of the functional annotation of the genes in this region, except for a monocot specific miRNA locus, osa-MIR437 of unknown function (Table 2 and Fig. 6).

\section{Discussion}

We conducted a phenotyping and the genome-wide association study of nine panicle morphological traits using 159 Vietnamese landrace accessions, to study the genetic basis of these traits and their putative interactions. The genotypic panel used had already been phenotyped for other traits (flowering time and root traits), and was deemed suitable for GWAS analysis as reported in [12, 22]. Our study allowed dissection of the various different components of panicle morphology relating to spikelet number variation and an evaluation of their contributions to this trait as well as to panicle size. This large-scale analysis was made possible by the use of an image-based software, which was especially useful for traits such as SBN, TBN and length traits [21]. A high heritability was observed for almost all morphological traits scored over the two years of field trials with the exception of TBN, as well as a good correlation between the two years, leading to the observation that the diversity of these traits was more under genetic control than under the influence of environmental conditions in this panel. In a similar way to results reported by [19] on an indica panel and by [20] on an indica and japonica panel, the main contributory component to variations observed in the Vietnamese panel is the variation of spikelet number ( $\mathrm{SpN})$, which is highly correlated with variation in secondary branch number (SBN) and, to a lesser extent, with variation in primary branch number (PBN). The high correlation of SBN with SpN was also reported in the context of bi-parental populations in a previous study [28]. The phenotypic variation of the panel was also analysed in the context of its genetic structure. The same phenotypic correlations were observed in the indica and japonica subpanels in our study. However, morphological differences were observed between these subpanels with, globally, larger panicle (i.e. high RL values) for the japonica than for the indica subpanel.

Overall, the level and nature of the observed correlations between traits show that variation of $\mathrm{SpN}$ relied more on 


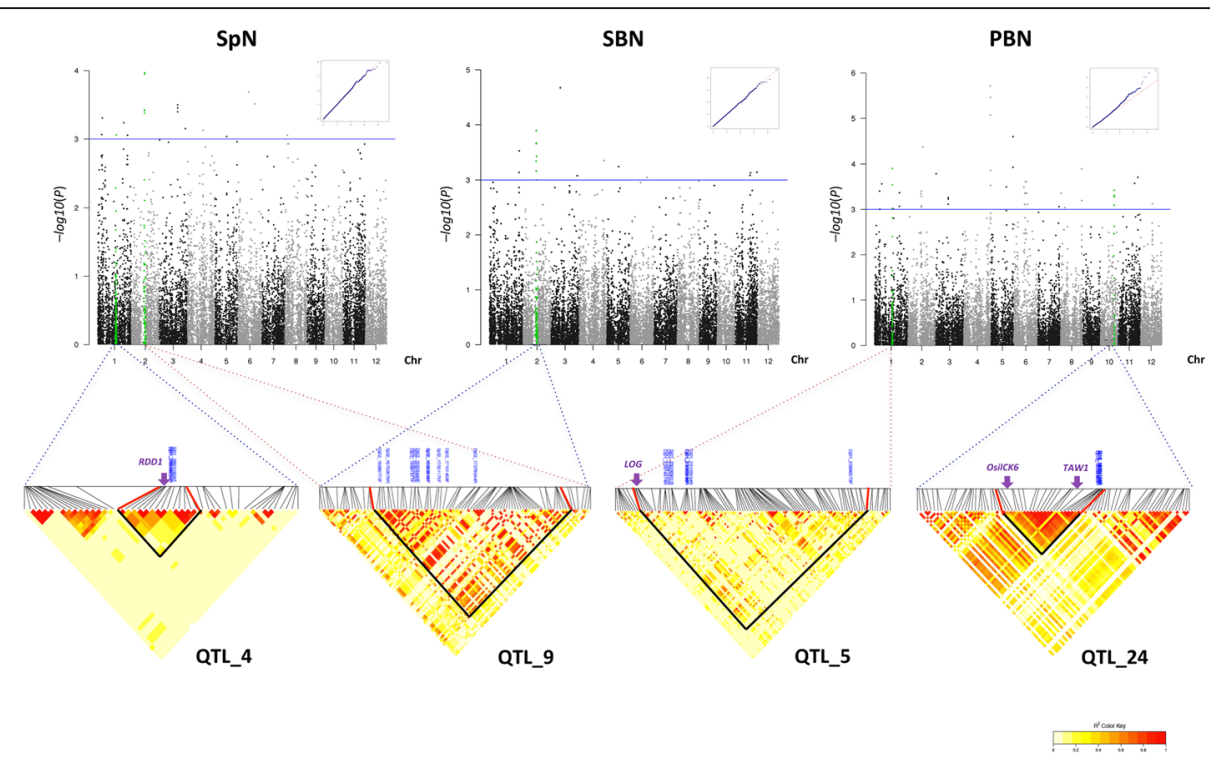

Fig. 6 Genome-wide association study of SpN, SBN and PBN traits in the full panel. From top to bottom, QQ plot, Manhattan plot over the 12 chromosomes, and Linkage Disequilibrium (LD) heatmap surrounding the peak in the 2014 experiment for spikelet number (SpN) with QTL_4 and QTL_9 on chromosome 1 and 2 respectively, secondary branch number (SBN) with QTL_9 on chromosome 2 and primary branch number (PBN) for QTL_5 and QTL_24 on chromosome 1 and 10 respectively. Arrows indicate the position of functionally characterized genes. Red and bold back lines on the LD heat maps delimit the LD block for the GWAS peak. The significant SNPs are labelled in blue in the LD heatmap

number traits rather than on length traits in this panel and that the number of branches is globally independent of their length of the axils. This independence is coherent considering the kinetic of panicle development in which the branching phase (i.e. axillary meristem establishment and outgrowth) and the elongation phase (i.e. axil elongation) are disconnected with the large axil elongation occurring after establishment of axillary meristems and out-growth [29].

Despite a high correlation between the SpN and SBN traits, only a single GWAS site with co-location of SpN and SBN was observed on chromosome 2 (see Fig. 6), as well as a single site for SpN and PBN on chromosome 1 (Table 2). Several GWAS sites related to SpN did not co-localised with other morphological traits suggesting that $\mathrm{SpN}$ can be dependent on other traits that the ones quantified in this study. Similarly, distinct GWAS sites were observed for PBN and SBN suggesting that the mechanisms related to their establishment and their functioning are related to different genetic determinants. All the axillary meristems from rachis axil will contribute to primary branches. In contrast, the axillary meristem fate from a primary branch will balance between branch meristem (i.e. leading to a secondary branch) and spikelet meristem (i.e. leading to a lateral spikelet; see Fig. 1) [29]. Consequently, the intra-specific diversity for spikelet number per panicle is, at least for a part, related to the balance in meristem fate control in the second order of branching for indeterminate (i.e. branch meristem) vs. determinate (i.e. spikelet meristem) fate. This is in agreement with $O$. sativa mutant analyses leading to a model showing that the diversity of panicle complexity is related to fine-tuning of fate changes between branch and spikelet meristems [30].

Overall, this genome-wide association study led to the identification of 29 sites detected over two years for the same trait(s), for all the morphological traits quantified in this study. However, only 12 QTLs were associated to more than two significant markers in the corresponding LD blocks. This analysis would benefit from a larger density of SNPs for the association study and confirmation of the QTLs with a low number of associations. Overall, the QTLs detected in this study were characterized by a good stability between years but a low effect (i.e. low significance values) as also reported in other studies [19]. This may be explained by the additive effect of numerous determinants with no widespread major QTL, supporting the notion of a complex genetic basis for the individual morphological traits of the panicle.

Even if similar phenotypic features were observed between the different panels, no clear co-location of the GWAS sites for the same trait(s) was observed between our GWA study and those from [18-20], (with the exception of some common sites between our study and that reported by [18]). This may be a consequence of differences or specificities of the panels used in the four studies. The panel used in our study focused on Vietnamese landraces from both indica and japonica and not on elite accessions, in contrast with the genotypic panels used in the other studies mentioned. Another non-exclusive explanation is the effect of environmental factors on morphological panicle traits, bearing in mind that 
the various studies mentioned were conducted in different places and conditions around the world. Environmental impact is clearly illustrated by [19] with only two QTLs detected over the two experiments in different field conditions and in [20] with 18 QTLs detected in at least two conditions of the three tested. Our study over two years was conducted in similar (but not identical) environments and 29 QTLs corresponding to 105 significant SNPs were detected over the two years.

Some of the QTLs identified in our study were associated with genes that control panicle development and architecture such as the TAWAWA1 (TAW1), LONELY GUY (LOG) and RICE DOF DAILY FLUCTUATIONS 1 (RDD1) genes [31-33], thus illustrating the validity of our study. The later three genes were isolated via mutants displaying altered panicle architecture and until now had not been shown to contribute to natural variation of panicle traits such as PBN for TAW1 and LOG (in QTL_24 and QTL_5 respectively) and $\mathrm{SpN}$ for $R D D 1$ (in QTL_4). The TAW1 gene, encoding a transcription factor of the ALOG family, was reported as a regulator of spikelet fate acquisition influencing global architecture (and more specifically branch number) through the timing of acquisition of the determinate fate [31]. Expression of the LOG gene, encoding a cytokinin-activating enzyme that functions in the final step of bioactive cytokinin synthesis was associated with panicle meristem activity [32]. Interestingly, the $R D D 1$ gene, encoding a DOF transcription factor, which promotes nutrient ion uptake and grain yield, is regulated by photoperiod and circadian clock [33]. This gene, which is expressed in mesophyll, illustrates the possibility of there being an indirect effect (nutrient uptake) on panicle architecture in a source-sink relationship. Another example of an indirect effect on panicle development is illustrated by the NARROW LEAF1 (NAL1) gene encoding a putative trypsin-like serine and cysteine protease, which affects vein patterning and polar auxin transport through cell proliferation [34]. The activity of NAL1 is associated with a larger panicle and higher yield and the gene is co-localized with various QTLs $[4,35,36]$. In a similar way, several GWAS-derived QTLs detected in the present work co-localized with QTLs from bi-parental population studies relating to other traits, such as number of vascular bundles, root branching, chlorophyll content or flag leaf length. We also observed co-localization of some of our GWAS QTLs with some identified in relation to root system architecture using the same genetic population [12]. These co-localized genomic positions might represent physically related but independent determinants; however they might also be an indicator of panicle trait being indirectly affected by other traits, such as root and/or leaf structure, through a source-sink relationship at plant level.

Other stable QTLs were found to be associated with functionally characterized genes that had not yet been reported as being involved in panicle architecture control but which may have an indirect effect on yield. These genes are involved in the processes of cell division (e.g. INHIBITORS OF CYCLIN-DEPENDENT KINASE 6, OsiICK6, associated with $\mathrm{PBN}$ ), flowering transition (e.g. FLAVIN-BINDING KELCH REPEAT F-Box 1, OsFKF1, associated with RL; CONSTANS-LIKE 4, OsCOL4, associated with $\mathrm{PBN}$ ), brassinosteroid pathway (e.g. INCREASED LEAF INCLINATION1, OsILI1, associated with PBintL) or cellulose content (e.g. OsCesA4/BC7 associated with $\mathrm{SpN}$ ) [37-41]. Other genes that co-localized with QTLs remain to be functionally characterized but may be of interest on the basis of their annotation and/or homology to key genes in other species, such as two genes relevant to auxin signaling: OsPILS2 and OsARF22, associated with PBN and PBL, corresponding respectively to a PIN-like and an Auxin Response Factor (ARF) gene [42, 43]; LOC_OsO1g42260, associated with $\mathrm{PBN}$ and $\mathrm{SpN}$, which encodes a putative homolog of LEUNIG (LUG), a transcriptional repressor in A. thaliana acting in floral organ identity specification [44]; and LOC_Os04g55070 associated with PBintL and corresponding to a paralog of the OsGA20ox2 / SEMIDWARF 1 (SD1) gene involved in the gibberellin biosynthesis pathway associated with plant growth and development [45].

In addition to the annotated genes, some microRNA loci of interest were found to be located in GWAS sites. The microRNA miR156 with a locus located in QTL_10 (PBN) is widely conserved in plants. It is known to be associated with panicle branching in rice through the targeting of transcripts of the SPL gene family, notably OSSPL14/IDEAL PLANT ARCHITECTURE (IPA) [46]. The microRNA miR160 located in QTL_13 (SpN) is reported to target Auxin Response Factors (ARFs) transcripts, notably OsARF18 which has pleiotropic effects on plant development and growth [47]. The microRNA miR162, which is widely conserved in plants, has a locus located in QTL_15 (RL). This microRNA is reported to be associated with the negative feedback regulation of Dicer-Like DCL1 transcripts in Arabidopsis by microRNA-guided mRNA degradation [48]. The microRNA miR171 targets transcripts of GRAS plant-specific transcription factor family associated with phase change from vegetative to reproductive development and shoot apical meristem maintenance in rice [49]. With the exception of miR156, none of these miRNAs has previously been implicated in the regulation of panicle development in rice, but on the basis of existing knowledge from rice and/or in other species, they may be good candidates. In addition to miR156, other microRNAs have been reported to be involved in panicle development, including miR172 which regulates APETALA2 (AP2) transcription factor genes [50,51], miR397 which regulates a laccase-like encoding gene [52] and miR396 which regulates a GROWTH REGULATING FACTOR 4 (OsGRF4) gene [53]. The later examples illustrate that microRNAs play an 
important role in panicle development and may be also considered as candidates for targeted breeding. In this context, it is interesting to note that studies of the genetic diversity of microRNA loci in O. sativa and its wild relative $O$. rufipogon revealed the presence of several loci showing significant signals for positive selection and/or potential domestication selection $[54,55]$.

As seen in other GWAS studies of panicle architecture [18-20], some important genes known to be involved in the regulation of inflorescence development such as $L A X P A N$ ICLE1 (LAX1), ABNORMAL PANICLE ORGANIZATION1 and 2 (APO1, APO2), MONOCULM1 (MOC1), DENSE AND ERECT PANICLE1 (DEP1) and SPL14/IPA1 [56, 57] were not detected in our study. Most of the aforementioned genes were characterized from mutants and play a very important role in panicle growth and development $[56,57]$. It is likely that any variation in these genes might tend to be eliminated in natural conditions if panicle growth were too severely affected. Alternatively sequence variations might occur at very low frequency and thus not be observed or filtered out in our analysis. Nevertheless a recent analysis revealed that a combination of favourable alleles for these genes might collectively produce a higher spikelet number in a specific panel, supporting the notion that there is a complex genetic network regulating this phenotypic feature [58].

Some stable QTLs related to number traits such as QTL_1 for PBN trait and QTL_9 for SpN and SBN traits were not observed to be associated with any functionally described genes. It will therefore be of interest to characterize the genes concerned in terms of their sequence and expression diversity in contrasting haplotypes of the relevant trait in order to identify candidates for functional validation. Another possible approach to investigate these statistically derived QTLs will be to develop bi-parental segregating populations for the validation and positional cloning of the QTL.

\section{Conclusions}

Using image-based analysis software it was possible to dissect different components of panicle architecture, revealing that variations in spikelet number per panicle relate mostly to variations in secondary branch number. The 29 stable panicle trait QTLs identified by GWAS in the present study will provide new information on novel genetic determinants of panicle architecture. All the QTLs identified in our study were characterised by small effects (i.e. low $p$-values), supporting the notion of complex genetic interactions for the control of individual components of panicle architecture. The overall structure of the gene network governing panicle traits is still far from resolved and only some of the previously identified players may be associated with the diversity studied here. The additive effects of these individual factors should be taken into consideration for breeding by the use of pyramidal or multigenic genome editing approaches $[58,59]$.

\section{Additional files}

Additional File 1: Table S1. Phenotypic and passport data of the 159 accessions of the Vietnamese rice landrace collection in 2014 and 2015. The indicated phenotypic data correspond to the average values for each trait per accession per year. Values relating to length are in cm. (XLS 157 kb)

Additional File 2: Figure S1. The phenotype variation of indica and japonica subpanels in 2014 and 2015. Primary branch number (PBN), secondary branch number (SBN), spikelet number (SpN), primary branch length $(\mathrm{PBL})$, primary internode length (PBintL), secondary branch length $(\mathrm{SBL})$, secondary internode length (SBintL), tertiary branch number (TBN) and rachis length (RL). The values relating to length are in $\mathrm{cm}$. Statistical significance ( $t$ test $p$ values) between the two subpanels for the different panicle morphological traits is indicated (PDF $378 \mathrm{~kb}$ )

Additional File 3: Figure S2. Correlation plots and PCA in the full panel, indica and japonica subpanels over the two years including flowering time (FT), plant height (PH), tiller number (TN) and efficient tiller number (eTN). (a) Correlation plots of the full panel in 2014 (left) and 2015 (right). (b) PCA of the full panel in 2014 (left) and 2015 (right). (c) Correlation plots of the indica (left) and the japonica (right) subpanels in 2014 (top) and 2015 (bottom). Primary branch number (PBN), secondary branch number (SBN), spikelet number $(\mathrm{SpN})$, primary branch length (PBL), primary internode length (PBintL), secondary branch length $(\mathrm{SBL})$, secondary internode length (SBintL), tertiary branch number (TBN) and rachis length (RL). (PDF $475 \mathrm{~kb}$ )

Additional File 4: Figure S3. Correlation between SpN and PBN traits in 2014 and 2015. In grey and orange are values for 2014 and 2015, respectively. The right panel indicates the value density for the two traits in 2014 (grey) and 2015 (orange). SpN: spikelet number. PBN: primary branch number. (PDF $123 \mathrm{~kb}$ )

Additional File 5: Figure S4 Heatmap clustering of the accessions related to panicle morphological traits in 2014 and 2015. In red and blue are the values for indica and japonica subpanels, respectively. Primary branch number (PBN), secondary branch number (SBN), spikelet number $(\mathrm{SpN})$, primary branch length $(\mathrm{PBL})$, primary internode length (PBintL), secondary branch length (SBL), secondary internode length (SBintL), tertiary branch number (TBN) and rachis length (RL). (PDF $271 \mathrm{~kb}$ )

Additional File 6: Table S2. List of the significant markers from GWAS in 2014 and 2015 in the full panel, the indica and the japonica subpanels. The position (POS, in bp) in the chromosome (CHR) of each marker (based on MSU7.0 O. sativa Nipponbare reference genome) is indicated. The $p$-values associated to the traits are indicated. The values in bold are below $1.10^{-4}$. Primary branch number (PBN), secondary branch number (SBN), spikelet number $(\mathrm{SpN})$, primary branch length $(\mathrm{PBL})$, primary internode length (PBintL), secondary branch length (SBL), secondary internode length (SBintL), tertiary branch number (TBN) and rachis length (RL). (XLS 151 kb)

Additional File 7: Figure S5. QQ plots and Manhattan plots for panicle morphological traits for 2014 and 2015 in the full panel, indica and japonica subpanels. (a, i) Rachis length (RL); (b, j) primary branch number (PBN); (c, k) secondary branch number (SBN); (d, I) spikelet number $(\mathrm{SpN})$; $(\mathbf{e}, \mathbf{m})$ primary branch length $(\mathrm{PBL}) ;(\mathbf{f}, \mathbf{n})$ primary branch internode length (PBintL); $(\mathbf{g}, \mathbf{o})$ secondary branch length $(\mathrm{SBL}) ;(\mathbf{h}, \mathbf{p})$ secondary branch internode length (SBintL) for 2014 and 2015, respectively (PDF 9355 kb)

Additional File 8: Table S3. Detailed information of the conserved GWAS sites over the two years (2014 and 2015). QTL_1 to QTL_29: conserved QTLs for the same trait(s) over the two years; QTL_30 to QTL_50: conserved QTL regions but for different traits between the two years; FP: full panel; SBN: secondary branch number; PBN: primary branch number; SpN: spikelet number; SBintL: secondary branch internode length; PBintL: primary branch internode length; PBL: primary branch length; SBL: secondary branch length; RL: Rachis length; PH: plant height; TN: tiller number; eTN: efficient tiller number. Locus-id: locus identification number in MSU7.0 (for coding genes) and mirBase V21 (for miRNAs); $\left(^{*}\right.$ ) Functionally characterized candidate genes in rice from OGRO and funRiceGenes databases (qtaro.abr.affrc.go.jp/ogro; funricegenes.ncpgr.cn). Genomic coordinates in red indicate genes that are located at a distance below $50 \mathrm{~kb}$ from the LD block of the corresponding QTL. (XLS $108 \mathrm{~kb}$ ) 


\section{Abbreviations}

CV: Coefficient of variation; eTN: Efficient tiller number average; FT: Flowering time after sowing; GWAS: Genome-wide association study; LD: Linkage disequilibrium; PBintL: Rachis internode length average; PBL: Primary branch length average; PBN: Primary branch number per panicle; PCA: Principal component analysis; PH: Plant height average; QTL: Quantitative trait locus; RL: Rachis length average; SBintL: Primary branch internode length average; SBL: Secondary branch length average; SBN: Secondary branch number per panicle; SNP: Single nucleotide polymorphism; SpN: Spikelet number per panicle; TBN: Tertiary branch number per panicle; TN: Tiller number average

\section{Acknowledgments}

The authors would like to thank Helene Adam, James Tregear and Thomas Harrop for critical reading of the paper and helpful advice, as well as Nguyen Van Toan, Vu Phuong Oanh and other undergraduate students for their valuable support in field care and panicle phenotyping.

\section{Funding}

In addition to institutional funding, this research is funded by Vietnam National Foundation for Science and Technology Development (NAFOSTED) under grant number 106-NN.02-2016.60, and was jointly supported by Agropolis Foundation (through the «Investissements d'avenir » programme (ANR-10-LABX-000101)) and Fondazione Cariplo under the reference ID EVOREPRICE 1201-004.

\section{Availability of data and materials}

The datasets supporting the results of this publication are included within the article and its Additional files.

\section{Authors' contributions}

GP, DNV, LM and JS designed and directed the research; TKN, KNG, CB and JS analysed the data; TKN and JS wrote the manuscript with input from all authors; TKN, HTL, NDT, MDC, HTG, PTPN, BI, TTTH, DBY, LTN and JS contributed to field experiments, panicle phenotyping and techniques. All authors edited and agreed on the final manuscript.

\section{Ethics approval and consent to participate}

Not applicable.

\section{Consent for publication}

Not applicable.

\section{Competing interests}

The authors declare that they have no competing interests.

\section{Publisher's Note}

Springer Nature remains neutral with regard to jurisdictional claims in published maps and institutional affiliations.

\footnotetext{
Author details

${ }^{1}$ LMI RICE, University of Montpellier, IRD, CIRAD, USTH, National Key Laboratory for Plant Cell Biotechnology, Agronomical Genetics Institute, Hanoi, Vietnam. '2UMR DIADE, University of Montpellier, IRD, Montpellier, France. ${ }^{3}$ CIRAD, UMR AGAP, University of Montpellier, INRA, Montpellier, France. ${ }^{4}$ Plant Resource Center, Hanoi, Vietnam. ${ }^{5}$ UMR LSTM, University of Montpellier, CIRAD, IRD, Montpellier, France. ${ }^{6}$ Present address: Plant Genetics Laboratory, National Institute of Genetics, Mishima, Japan. ${ }^{7}$ Present address: Department of Molecular Biology, Palacký University, Olomouc, Czech Republic.
}

Received: 19 March 2018 Accepted: 26 October 2018 Published online: 14 November 2018

\section{References}

1. Xing Y, Zhang Q. Genetic and molecular bases of rice yield. Annu Rev Plant Biol. 2010;61:421-42.

2. Ikeda M, Miura K, Aya K, Kitano H, Matsuoka M. Genes offering the potential for designing yield-related traits in rice. Curr Opin Plant Biol. 2013;16:1-8.

3. Bai X, Wu B, Xing Y. Yield-related QTLs and their applications in rice genetic improvement. J Integr Plant Biol. 2012:54:300-11.
4. Fujita D, Trijatmiko KR, Tagle AG, Sapasap MV, Koide Y, Sasaki K, et al. NAL 1 allele from a rice landrace greatly increases yield in modern indica cultivars. Proc Natl Acad Sci U S A. 2013;110:20431-6.

5. Takai T, Adachi S, Taguchi-Shiobara F, Sanoh-Arai Y, Iwasawa N, Yoshinaga S, et al. A natural variant of NAL1, selected in high-yield rice breeding programs, pleiotropically increases photosynthesis rate. Sci Rep. 2013;3:2149.

6. Zhu C, Gore M, Buckler ES, Yu J. Status and prospects of association mapping in plants. Plant Gen. 2008;1:5-20.

7. Huang X, Wei X, Sang T, Zhao Q, Feng Q, Zhao Y, et al. Genome-wide association studies of 14 agronomic traits in rice landraces. Nat Genet. 2010;42:961-7.

8. Chen W, Gao Y, Xie W, Gong L, Lu K, Wang W, et al. Genome-wide association analyses provide genetic and biochemical insights into natural variation in rice metabolism. Nat Genet. 2014;46:714-21.

9. Yonemaru J-I, Mizobuchi R, Kato H, Yamamoto T, Yamamoto E, Matsubara K, et al. Genomic regions involved in yield potential detected by genomewide association analysis in Japanese high-yielding rice cultivars. BMC Genomics. 2014;15:346.

10. Magwa RA, Zhao H, Xing Y. Genome-wide association mapping revealed a diverse genetic basis of seed dormancy across subpopulations in rice (Oryza sativa L.). BMC Genet. 2016;17:28.

11. Matsuda F, Nakabayashi R, Yang Z, Okazaki Y, Yonemaru J-I, Ebana K, et al. Metabolome-genome-wide association study dissects genetic architecture for generating natural variation in rice secondary metabolism. Plant J. 2014; 81:13-23.

12. Phung NTP, Mai CD, Hoang GT, Truong HTM, Lavarenne J, Gonin M, et al. Genome-wide association mapping for root traits in a panel of rice accessions from Vietnam. BMC Plant Biol. 2016;16:64.

13. Kumar V, Singh A, Mithra SV, Krishnamurthy SL, Parida SK, Jain S, et al. Genome-wide association mapping of salinity tolerance in rice (Oryza sativa). DNA Res. 2015;22:133-45.

14. Famoso AN, Zhao K, Clark RT, Tung C-W, Wright MH, Bustamante C, et al. Genetic architecture of aluminum tolerance in rice (Oryza sativa) determined through genome-wide association analysis and QTL mapping. PLoS Genet. 2011;7:e1002221.

15. Begum H, Spindel JE, Lalusin A, Borromeo T, Gregorio G, Hernandez J, et al. Genome-wide association mapping for yield and other agronomic traits in an elite breeding population of tropical rice (Oryza sativa). PLoS One. 2015; 10:e0119873.

16. Huang X, Zhao Y, Wei X, Li C, Wang A, Zhao Q, et al. Genome-wide association study of flowering time and grain yield traits in a worldwide collection of rice germplasm. Nat Genet. 2011:44:32-9.

17. Zhao K, Tung C-W, Eizenga GC, Wright MH, Ali ML, Price AH, et al. Genomewide association mapping reveals a rich genetic architecture of complex traits in Oryza sativa. Nat Commun. 2011;2:467.

18. Crowell S, Korniliev P, Falcão A, Ismail A, Gregorio G, Mezey J, et al. Genome-wide association and high-resolution phenotyping link Oryza sativa panicle traits to numerous trait-specific QTL clusters. Nat Commun. 2016;7:10527

19. Rebolledo MC, Peña AL, Duitama J, Cruz DF, Dingkuhn M, Grenier C, et al. Combining image analysis, genome wide association studies and different field trials to reveal stable genetic regions related to panicle architecture and the number of spikelets per panicle in rice. Front Plant Sci. 2016;7:122-12.

20. Bai X, Zhao H, Huang Y, Xie W, Han Z, Zhang B, et al. Genome-wide association analysis reveals different genetic control in panicle architecture between indica and japonica rice. Plant Gen. 2016. https://doi.org/10.3835/ plantgenome2015.11.0115.

21. AL-Tam F, Adam H, Anjos AD, Lorieux M, Larmande $P$, Ghesquière $A$, et al. P-TRAP: a panicle trait phenotyping tool. BMC Plant Biol. 2013;13:122.

22. Phung NTP, Mai DC, Mournet P, Frouin J, Droc G, Ta KN, Jouannic S, et al. 2014 Characterization of a panel of Vietnamese rice varieties using DArT and SNP markers for association mapping purposes. BMC Plant biol. 2014;14:371.

23. Fukuoka S, Alpatyeva NV, Ebana K, Luu NT, Nagamine T. Analysis of Vietnamese rice germplasm provides an insight into japonica rice differentiation. Plant Breed. 2003:122:497-502.

24. Pritchard JK, Stephens M, Donnelly P. Inference of population structure using multilocus genotype data. Genetics. 2000;155:945-59.

25. Patterson HD, Williams ER. A new class of resolvable incomplete block designs. Biometrika. 1976:63:83-92.

26. Yu J, Pressoir G, Briggs WH, Vroh Bi I, Yamasaki M, Doebley JF, et al. A unified mixed-model method for association mapping that accounts for multiple levels of relatedness. Nat Genet. 2006;38:203-8. 
27. Bradbury PJ, Zhang Z, Kroon DE, Casstevens TM, Ramdoss Y, Buckler E. TASSEL: software for association mapping of complex traits in diverse samples. Bioinformatics. 2007;23:2633-5.

28. Mei HW, Xu JL, Li ZK, Yu XQ, Guo LB, Wang YP, et al. QTLs influencing panicle size detected in two reciprocal introgressive line (IL) populations in rice (Oryza sativa L.). Theor Appl Genet. 2006;112:648-56.

29. Itoh J, Nonomura K, Ikeda K, Yamaki S, Inukai Y, Yamagishi H, et al. Rice plant development: from zygote to spikelet. Plant Cell Physiol. 2005;46:23-47.

30. Kyozuka J, Tokunaga H, Yoshida A. Control of grass inflorescence form by the fine-tuning of meristem phase change. Curr Opin Plant Biol. 2014;17:110-5.

31. Yoshida A, Sasao M, Yasuno N, Takagi K, Daimon Y, Chen R, et al. TAWAWA1, a regulator of rice inflorescence architecture, functions through the suppression of meristem phase transition. Proc Natl Acad Sci U S A. 2013;110:767-72.

32. Kurakawa T, Ueda N, Maekawa M, Kobayashi K, Kojima M, Nagato Y, et al. Direct control of shoot meristem activity by a cytokinin-activating enzyme. Nature. 2007:445:652-5.

33. Iwamoto $\mathrm{M}$, Tagiri $\mathrm{A}$. MicroRNA-targeted transcription factor gene RDD1 promotes nutrient ion uptake and accumulation in rice. Plant J. 2016;85: 466-77.

34. Jiang D, Fang J, Lou L, Zhao J, Yuan S, Yin L, Sun W, Peng L, Guo B, Li X Characterization of a null allelic mutant of the rice NAL1 gene reveals its role in regulating cell division. PLoS One. 2015;10:e0118169.

35. Adriani DE, Dingkuhn M, Dardou A, Adam H, Luquet D, Lafarge T. Rice panicle plasticity in near isogenic lines carrying a QTL for larger panicle is genotype and environment dependent. Rice (N Y). 2016;9:28.

36. Zhang GH, Li SY, Wang L, Ye WJ, Zeng DL, Rao YC, Peng YL, Hu J, Yang YL, Xu J, Ren DY, Gao ZY, Zhu L, Dong GJ, Hu XM, Yan MX, Guo LB, Li CY, Qian Q. LSCHL4 from japonica cultivar, which is allelic to NAL1, increases yield of indica super rice 93-11. Mol Plant. 2014;7:1350-64.

37. Yang $R$, Tang $Q$, Wang H, Zhang X, Pan G, Wang H, Tu J. Analyses of two rice (Oryza sativa) cyclin-dependent kinase inhibitors and effects of transgenic expression of OsilCK6 on plant growth and development. Ann Bot. 2011;107:1087-101.

38. Han SH, Yoo SC, Lee BD, An G, Paek NC. Rice FLAVIN-BINDING, KELCH REPEAT, F-BOX 1 (OsFKF1) promotes flowering independent of photoperiod. Plant Cell Environ. 2015:38:2527-40.

39. Lee YS, Jeong DH, Lee DY, Yi J, Ryu CH, Kim SL, et al. OsCOL4 is a constitutive flowering repressor upstream of Ehd 1 and downstream of OsphyB. Plant J. 2010;63:18-30.

40. Zhang LY, Bai MY, Wu J, Zhu JY, Wang H, Zhang Z, et al. Antagonistic HLH/ bHLH transcription factors mediate brassinosteroid regulation of cell elongation and plant development in rice and Arabidopsis. Plant Cell. 2009; 21:3767-80

41. Tanaka K, Murata K, Yamazaki M, Onosato K, Miyao A, Hirochika H. Three distinct rice cellulose synthase catalytic subunit genes required for cellulose synthesis in the secondary wall. Plant Physiol. 2003;133:73-83.

42. Mohanta TK, Mohanta N, Bae H. Identification and expression analysis of PIN-like (PILS) gene family of rice treated with auxin and cytokinin. Genes. 2015:6:622-40

43. Wang D, Pei K, Fu Y, Sun Z, Li S, Liu H, et al. Genome-wide analysis of the auxin response factors (ARF) gene family in rice (Oryza sativa). Gene. 2007; 394:13-24.

44. Sridhar W, Surendrarao A, Gonzalez D, Conlan RS, Liu Z. Transcriptional repression of target genes by LEUNIG and SEUSS, two interacting regulatory proteins for Arabidopsis flower development. Proc Natl Acad Sci U S A. 2004;101:11494-9.

45. Spielmeyer W, Ellis MH, Chandler PM. Semidwarf (sd-1), "green revolution" rice, contains a defective gibberellin 20-oxidase gene. Proc Natl Acad Sci U S A. 2002;99:9043-8

46. Jiao Y, Wang Y, Xue D, Wang J, Yan M, Liu G, et al. Regulation of OsSPL14 by OsmiR156 defines ideal plant architecture in rice. Nat Genet. 2010;42:541-4.

47. Huang J, Li Z, Zhao D. Deregulation of the osmiR160 target gene OsARF18 causes growth and developmental defects with an alteration of auxin signaling in rice. Sci Rep. 2016;6:29938.

48. Xie Z, Kasschau KD, Carrington JC. Negative feedback regulation of dicerLike1 in Arabidopsis by microRNA-guided mRNA degradation. Curr Biol. 2003;13:784-9.

49. Fan T, Li X, Yang W, Xia K, Ouyang J, Zhang M. Rice Osa-miR171c mediates phase change from vegetative to reproductive development and shoot apical meristem maintenance by repressing four OsHAM transcription factors. PLoS One. 2015;10:e0125833.
50. Zhu QH, Upadhyaya NM, Gubler F, Helliwell CA. Over-expression of miR172 causes loss of spikelet determinacy and floral organ abnormalities in rice (Oryza sativa). BMC Plant Biol. 2009;9:149.

51. Wang $L$, Sun $S$, Jin J, Fu D, Yang $X$, Weng $X$, et al. Coordinated regulation of vegetative and reproductive branching in rice. Proc Natl Acad Sci U S A. 2015;112:15504-9.

52. Zhang YC, Yu Y, Wang CY, Li ZY, Liu Q, Xu J, et al. Overexpression of microRNA OsmiR397 improves rice yield by increasing grain size and promoting panicle branching. Nat Biotechnol. 2013;31:848-52.

53. Li S, Gao F, Xie K, Zeng X, Cao Y, Zeng J, et al. The OsmiR396c-OsGRF4OsGIF1 regulatory module determines grain size and yield in rice. Plant Biotechnol J. 2016;14:2134-46.

54. Wang $Y$, Shen D, Bo S, Chen H, Zheng J, Zhu QH, et al. Sequence variation and selection of small RNAs in domesticated rice. BMC Evol Biol. 2010;10:119.

55. Wang Y, Bai X, Yan C, Gui Y, Wei X, Zhu QH, et al. Genomic dissection of small RNAs in wild rice (Oryza rufipogon): lessons for rice domestication. New Phytol. 2012;196:914-25.

56. Wang Y, Li J. Branching in rice. Curr Opin Plant Biol. 2011;14:94-9.

57. Teo ZW, Song S, Wang YQ, Liu J, Yu H. New insights into the regulation of inflorescence architecture. Trends Plant Sci. 2014;19:158-65.

58. Jang $S$, Lee $Y$, Lee $G$, Seo J, Lee D, Yu Y, et al. Association between sequence variants in panicle development genes and the number of spikelets per panicle in rice. BMC Genet. 2018;19:5.

59. Li M, Li X, Zhou Z, Wu P. Fang M, Pan X, et al. Reassessment of the four yield-related genes Gn1a, DEP1, GS3, and IPA1 in Rice using a CRISPR/Cas9 system. Front Plant Sci. 2016;7:377.
Ready to submit your research? Choose BMC and benefit from:

- fast, convenient online submission

- thorough peer review by experienced researchers in your field

- rapid publication on acceptance

- support for research data, including large and complex data types

- gold Open Access which fosters wider collaboration and increased citations

- maximum visibility for your research: over $100 \mathrm{M}$ website views per year

At $\mathrm{BMC}$, research is always in progress.

Learn more biomedcentral.com/submissions 